\title{
Composição corporal de cálcio e fósforo do útero gestante e da glândula mamária de ovelhas Santa Inês
}

[Calcium and phosphorus content in gravidic uterus and mammary gland of Santa Inês breed ewes]

\author{
F.A. Souza ${ }^{1}$, I. Borges ${ }^{2,5}$, G.L. Macedo Junior ${ }^{3}$, V.B. Silva ${ }^{4}$, A.L.C.C. Borges ${ }^{2,5}$, E.O.S. Saliba ${ }^{2,5}$ \\ ${ }^{1}$ Aluno de pós-graduação - Escola de Medicina - Universidade Federal de Minas Gerais - Belo Horizonte, MG \\ ${ }^{2}$ Escola de Veterinária - Universidade Federal de Minas Gerais - Belo Horizonte, MG \\ ${ }^{3}$ Universidade Federal de Uberlândia - Uberlândia, MG \\ ${ }^{4}$ Aluna de pós-doutorado - Universidade Federal de Alagoas - Bolsista Capes \\ ${ }^{5}$ Bolsista de produtividade do CNPq
}

\begin{abstract}
RESUMO
Ovelhas Santa Inês com gestação simples e múltipla foram alimentadas com dietas formuladas para atender a $100 \%$ - grupo não restrito - e $85 \%$ - grupo restrito - das suas necessidades de energia metabolizável (EM) e proteína bruta (PB). Os animais foram abatidos aos 140 dias de gestação para a retirada do útero da glândula mamária e do útero gestante, os quais foram analisados para o conteúdo de cálcio e de fósforo em função dos tratamentos. A restrição nutricional não influenciou as concentrações de cálcio e fósforo no feto e no útero gestante. Foi observado um aumento de 11,68 gramas de cálcio e de 6,37 gramas de fósforo para cada quilograma de massa fetal produzido aos 140 dias de gestação. As concentrações de cálcio e de fósforo do útero e das membranas foram mais baixas nas ovelhas gestantes submetidas à restrição nutricional. $\mathrm{O}$ número de fetos e o manejo nutricional não resultaram em alterações na concentração nem no conteúdo de cálcio e fósforo nos fluidos fetais. O conteúdo de cálcio e de fósforo no útero gestante teve como principal fator de variação o tamanho da massa fetal produzida.
\end{abstract}

Palavras-chave: ovelha, cálcio, fósforo, gestação, feto

\begin{abstract}
Santa Inês breed ewes with single and multiple gestations were fed diets to meet 100\% - Group ad libitum and $85 \%$ - restricted group - of metabolizable energy (ME) and crude protein $(C P)$ needs. The animals were slaughtered at 140 days of gestation for the removal of the gravidic uterus and mammary gland which were analyzed for calcium and phosphorus content. The nutritional restriction did not influence the calcium and phosphorus concentrations in the fetus and the pregnant uterus. The calcium and phosphorus content in the gravidic utero increased 11.68 grams and 6.37 grams for each kilogram of fetal mass produced at 140 days of gestation respectively. The calcium and phosphorus concentration in the uterus and membranes were lower in pregnant ewes subjected to nutritional restriction. The number of fetuses and nutritional management did not result in changes in the concentration or amount of calcium and phosphorus in fetal fluids. The calcium and phosphorus content in the gravidic uterus had the fetal weight as main variation factor.
\end{abstract}

Keyword: ewes, calcium, phosphorus, pregnancy, fetuses

\section{INTRODUÇÃO}

Necessidade nutricional de cálcio e fósforo de ovelhas ao longo do período de gestação é definida como a quantidade retida no feto e em tecidos anexos - placenta, útero e fluidos fetais , sendo o feto responsável por $97 \%$ e $80 \%$ do aumento da demanda de cálcio e fósforo durante a gestação, respectivamente (Grace et al., 1986).

Os atuais sistemas de recomendação de exigências de cálcio e de fósforo NRC (Nutrient..., 2007); CSIRO (Nutrient..., 2007); AFRC (1991) baseiam-se nos valores

Recebido em 30 de agosto de 2011 
apresentados pelo ARC (Nutrient.., 1980) o qual estima conteúdo de cálcio e de fósforo igual a 52 e 28 gramas, respectivamente, para fetos pesando $4 \mathrm{~kg}$ aos 147 dias de gestação. Estes valores, entretanto, podem não ser verdadeiros para fetos Santa Inês, os quais apresentam peso médio ao nascimento de 3,2kg (Quesada et al., 2002; Mexia et al., 2004; Rocha et al., 2009). Não obstante o peso ao nascimento, a proporção osso:músculo no feto define em grande parte a quantidade diária destes minerais retida, sendo que fetos com menor proporção tendem a ter um menor conteúdo de cálcio e de fósforo retido do corpo.

Este trabalho objetivou avaliar o efeito do tipo de gestação sobre a composição de cálcio e de fósforo do útero gestante e da glândula mamária de ovelhas Santa Inês.

\section{MATERIAL E MÉTODOS}

Foram analisadas, para o conteúdo de cálcio e de fósforo, amostras dos úteros gestantes e de glândulas mamárias de ovelhas não gestantes e gestantes de um, dois e três fetos oriundas de um experimento realizado entre os anos de 2006 e 2008 para determinação de exigências de energia e proteína em ovelhas gestantes (Macedo Junior, 2008).

Utilizaram-se 38 úteros gestantes e glândulas mamárias de ovelhas da raça Santa Inês, adultas gestantes de 140 dias. Os grupos experimentais foram formados por: 10 úteros e glândulas mamárias oriundos de ovelhas não gestantes, as quais serviram como grupo de referência; sete úteros gestantes e glândulas mamárias oriundos de ovelhas com um feto; 13 úteros gestantes e glândulas mamárias oriundos de ovelhas com dois fetos; e oito úteros gestantes e glândulas mamarias oriundos de ovelhas com três fetos.

As ovelhas foram acasaladas com reprodutores Santa Inês, e a determinação da prenhez e do número de fetos foi realizada por meio de ultrassom, 30 dias após o fim do período de estação de monta. Os animais gestando um e dois fetos foram distribuídos em um delineamento inteiramente ao acaso, em arranjo fatorial $2 \times 3$ (manejo nutricional $x$ número de fetos). Devido ao risco de problemas gestacionais, os animais com gestação tripla não foram submetidos à restrição nutricional.
Todas as ovelhas foram adaptadas às instalações e aos manejos por um período de 15 dias. Os animais foram alimentados com uma dieta composta por feno de tifton (Cynodon spp.), farelo de milho (Zea Mays), farelo de soja (Glycine Max) e calcário, balanceada para atender as necessidades nutricionais de proteína bruta (PB) e nutrientes digestíveis totais (NDT) em $100 \%$ - grupo ad libtum (NR) - e $85 \%$ grupo restrito $(\mathrm{R})$.

Após o fim do período de adaptação, os animais do grupo de referência foram sacrificados para a retirada do útero gestante e da glândula mamária. Os úteros gestantes, separados da vagina na cérvix após sangria e evisceração, foram pesados cheios e, em seguida, divididos em três partes para análise do conteúdo de cálcio e fósforo: (1) fluidos fetais, (2) feto e (3) útero, membranas e cotilédones. Analisaram-se amostras pré-secas em estufa de circulação forçada de ar a $55^{\circ} \mathrm{C}$ e pré-desengorduradas e moídas a $1 \mathrm{~mm}$ (Association..., 1985). A solução mineral foi aberta por meio de via úmida utilizando-se uma solução nitroperclórica composta por $75 \%$ de ácido nítrico p.a e $25 \%$ de ácido perclórico p.a (v/v). A solução mineral foi analisada para o conteúdo de cálcio por meio de espectrometria de absorção atômica e para o conteúdo de fósforo por meio de métodos colorimétricos. O conteúdo de cálcio e de fósforo na matéria pré-seca foi convertido novamente para matéria natural anterior à análise de variância.

As médias de cada tratamento foram comparadas por meio do test SNK (Student- NewmanKeuss), com probabilidade para o erro igual a $5 \%$. Foi avaliado o efeito de manejo e número de fetos para úteros gestantes e glândulas mamárias de ovelhas gestando um e dois fetos. Para amostras oriundas de ovelhas gestando um, dois e três fetos, utilizaram-se para comparação apenas os dados oriundos de ovelhas que não foram submetidas à restrição nutricional.

\section{RESULTADO E DISCUSSÃO}

$\mathrm{O}$ peso dos componentes do útero gestante em função do número de fetos gestados é apresentado na Tab. 1. O peso do útero gravídico elevou-se com o aumento do número de fetos, o qual se deve ao aumento da massa de todos os seus componentes - útero e membranas, feto e fluidos gestacionais. $\mathrm{O}$ peso do útero/membranas 
elevou-se 1,8 vezes nos animais com gestação trigemelar em relação aos animais de gestação simples. A massa fetal praticamente dobrou entre os animais com gestação simples e tripla e respondeu por aproximadamente $60 \%$ do peso do útero gestante.

Tabela 1. Peso em gramas do feto, útero e fluidos fetais em função do número de fetos

\begin{tabular}{cccccc} 
& \multicolumn{3}{c}{ Número de fetos } & & CV \\
\cline { 2 - 6 } & 0 & 1 & 2 & 3 & $\%$ \\
\hline Útero & $93,27 \mathrm{c}$ & $1.306,66 \mathrm{~b}$ & $1.889,25 \mathrm{~b}$ & $2.429,88 \mathrm{a}$ & 21,77 \\
Feto & $* *$ & $3.476,66 \mathrm{~b}$ & $6.677,12 \mathrm{a}$ & $8.427,50 \mathrm{a}$ & 22,56 \\
Fluido placentário $(\mathrm{mL})$ & $* *$ & $736,66 \mathrm{~b}$ & $1.688,75 \mathrm{ab}$ & $2.266,25 \mathrm{a}$ & 44,48 \\
Útero gestante & $* *$ & $5.519,98 \mathrm{~b}$ & $10.239,86 \mathrm{a}$ & $13.194,18 \mathrm{a}$ & 24,32 \\
\hline
\end{tabular}

O tipo de gestação elevou a concentração e o conteúdo de cálcio no útero das ovelhas (Tab. 2). Este efeito está relacionado ao aumento da massa do tecido uterino e ao aumento das membranas placentárias. A concentração de fósforo em gramas por quilograma no tecido uterino não foi afetada pelo tipo de gestação, sendo o aumento da quantidade total observada resultado do aumento da massa uterina observada. Em ovinos, o desenvolvimento placentário é correlacionado com o peso ao nascimento (Kelly,1992) e com o tipo de gestação (simples ou dupla) (Ocak et al., 2009).

Tabela 2. Concentração e conteúdo de cálcio $(\mathrm{Ca})$ e de fósforo $(\mathrm{P})$ de ovelhas Santa Inês gestantes com 1 , 2 e 3 fetos

\begin{tabular}{|c|c|c|c|c|c|}
\hline & & & nero de fe & & \\
\hline & 0 & 1 & 2 & 3 & $\mathrm{CV} \%$ \\
\hline Útero e membranas & & & & & \\
\hline $\mathrm{Ca}(\mathrm{g} / \mathrm{Kg})$ & 0,18 & 0,42 & ,72 & 0,97 & 55,88 \\
\hline $\mathrm{Ca}(\mathrm{g})$ & $0,016 \mathrm{c}$ & $0,54 \mathrm{~b}$ & $1,36 \mathrm{~b}$ & $2,12 \mathrm{a}$ & 17,66 \\
\hline $\mathrm{P}(\mathrm{g} / \mathrm{Kg})$ & 0,39 & 0,49 & 0,46 & 0,54 & 20,45 \\
\hline $\mathrm{P}(\mathrm{g})$ & $0,035 \mathrm{c}$ & $0,63 \mathrm{~b}$ & $0,87 \mathrm{~b}$ & $1,30 \mathrm{a}$ & 24,28 \\
\hline Feto & & & & & \\
\hline $\mathrm{Ca}(\mathrm{g} / \mathrm{Kg})$ & ------ & 15,45 & 13,23 & 12,43 & 24,22 \\
\hline $\mathrm{Ca}(\mathrm{g})$ & ----- & $66,19 \mathrm{c}$ & $86,91 \mathrm{~b}$ & $106,40 \mathrm{a}$ & 34,67 \\
\hline$P(g / K g)$ & ------ & 7,85 & 6,87 & 6,68 & 17,96 \\
\hline$P(g)$ & ------ & $33,03 \mathrm{c}$ & $42,26 \mathrm{~b}$ & $57,18 \mathrm{a}$ & 30,90 \\
\hline Fluido fetal & ------ & & & & \\
\hline $\mathrm{Ca}(\mathrm{g} / \mathrm{Kg})$ & ------ & 2,87 & 1,64 & 1,71 & 48,85 \\
\hline $\mathrm{Ca}(\mathrm{g})$ & ------ & 0,12 & 0,16 & 0,21 & 42,15 \\
\hline $\mathrm{P}(\mathrm{g} / \mathrm{Kg})$ & ------ & 0,074 & 0,022 & 0,037 & 31,00 \\
\hline$P(g)$ & ------ & 0,057 & 0,037 & 0,083 & 58,45 \\
\hline Útero gestante & ------ & & & & \\
\hline $\mathrm{Ca}(\mathrm{g} / \mathrm{Kg})$ & ----- & 10,60 & 8,81 & 8,15 & 24,60 \\
\hline $\mathrm{Ca}(\mathrm{g})$ & ------ & $67,19 \mathrm{c}$ & $87,88 \mathrm{~b}$ & $18,73 \mathrm{a}$ & 33,61 \\
\hline$P(g / K g)$ & ------ & 5,44 & 4,59 & 4,38 & 17,72 \\
\hline$P(g)$ & ------ & $33,72 \mathrm{c}$ & $46,17 \mathrm{~b}$ & $58,17 \mathrm{a}$ & 30,38 \\
\hline $\mathrm{Ca}$ feto: Ca útero gestante (\%) & ------ & 98,46 & 98,36 & 97,34 & 1,92 \\
\hline $\mathrm{P}$ feto : $\mathrm{P}$ útero gestante $(\%)$ & ------ & 97,90 & 97,97 & 97,40 & 0,84 \\
\hline Ca: $P$ feto & ----- & 1,95 & 1,90 & 1,85 & 10,67 \\
\hline $\mathrm{Ca}: \mathrm{P}$ útero gestante & ------ & 1,94 & 1,88 & 1,86 & 10,89 \\
\hline Glândula mamária & ------ & & & & \\
\hline $\mathrm{Ca}(\mathrm{g} / \mathrm{Kg})$ & ----- & 5,89 & 4,05 & 3,58 & 50,74 \\
\hline $\mathrm{Ca}(\mathrm{g})$ & ------ & 3,53 & 2,75 & 3,15 & 67,45 \\
\hline $\mathrm{P}(\mathrm{g} / \mathrm{Kg})$ & ------ & 7,94 & 6,33 & 6,09 & 1,95 \\
\hline$P(g)$ & ------ & $4,66 \mathrm{~b}$ & $4,63 \mathrm{~b}$ & $4,93 \mathrm{a}$ & 3,52 \\
\hline
\end{tabular}


A concentração de cálcio e de fósforo do feto não foi afetada pela restrição ou pelo número de fetos. O conteúdo de cálcio e de fósforo foi maior nos fetos de ovelhas gestantes com três e dois fetos do que nas ovelhas com gestação simples. Isto se deve ao fato de os fetos oriundos de gestação múltipla terem sido analisados em conjunto e de maior massa fetal ser produzida neste tipo de gestação. Grace et al. (1986) encontraram para ovelhas New Zealand Romney, aos 143 dias de gestação, conteúdo de cálcio e de fósforo igual a 74,23 e 36,24 gramas em fetos de gestação simples e 108,8 e 50,66 gramas para fetos de gestação dupla. As diferenças observadas podem ser atribuídas a diferenças na massa fetal produzida, uma vez que, no experimento supracitado, o peso do feto era de $5,34 \mathrm{~kg}$ para fetos simples e $8,232 \mathrm{~kg}$ para ovelhas de gestação dupla contra o peso de $3,58 \mathrm{~kg}$ e $6,42 \mathrm{~kg}$ para fetos de gestação simples e dupla aos 140 dias observados neste experimento. A concentração de cálcio e de fósforo no feto encontrada por estes autores na matéria natural aos 143 dias foi de $13,8 \mathrm{~g} / \mathrm{kg}$ e $6,7 \mathrm{~g} / \mathrm{kg}$ de feto gestado respectivamente estando de acordo com os valores encontrados neste trabalho.

Análise de regressão mostrou haver relação linear entre peso total do(s) feto(s) gestado(s) aos 140 dias e conteúdo de cálcio e de fósforo retido, o qual pode ser predito pela equação $(\mathrm{P}<0,01)$ :

C $a_{\text {feto }}=9,95+11,68 P V_{\text {feto }}(1)$,

$P_{\text {feto }}=4,45+6,37 P V_{\text {feto }} \quad(2)$,

em que:

$\mathrm{Ca}_{\text {feto }}=$ conteúdo de cálcio no feto em gramas $(\mathrm{g})$

$\mathrm{P}_{\text {feto }}=$ conteúdo de fósforo no feto em gramas $(\mathrm{g})$

$\mathrm{PV}_{\text {feto }}=$ peso do feto aos 140 dias de gestação em quilogramas $(\mathrm{kg})$.

Baseado nestas equações, um feto de $4 \mathrm{~kg}$ aos 140 dias de gestação contém, em média, 56,67 gramas de cálcio e 29,93 gramas de fósforo, contra um conteúdo igual a 54,06 gramas para o cálcio e 23,95 gramas para o fósforo predito pelo ARC (Nutrient..., 1980) .

Houve efeito de interação gestação versus manejo nutricional para o conteúdo de cálcio nas amostras de útero e membranas, sendo menores para as ovelhas gestantes submetidas à restrição nutricional (Tab. 3). O conteúdo de fósforo não foi afetado pelo manejo nutricional e pelo número de fetos, porém ovelhas vazias apresentaram as menores concentrações/ conteúdos, o que se atribui ao fato de nesse estádio não haver membranas, carúnculas e resíduos de fluidos fetais presentes na gestação.

A concentração de cálcio dos fluidos fetais foi afetada pelo manejo nutricional e pelo número de fetos, sendo menores valores observados nas ovelhas com gestação múltipla e nas ovelhas que não sofreram restrição. As amostras de fluido fetal foram compostas pelos líquidos amniótico e alantoide. Segundo Pearson e Mellor (1977), a concentração de cálcio no líquido alantoide de caprinos aos 140 dias é igual a 1,83mMol (aprox. 0,073 gramas). Alexander et al. (1958) encontraram concentração de fósforo no líquido amniótico igual a $2,7 \mathrm{mg} / 100 \mathrm{~mL}$ e no fluido alantoide 7,3mg/100mL - aprox. 0,013 gramas e 0,035 gramas, respectivamente. No presente trabalho, o conteúdo de cálcio no fluido fetal oriundo de gestação gemelar foi o dobro do conteúdo observado em gestação simples - 0,153 vs. 0,070 gramas -, e nenhum efeito dos tratamentos foi observado para o conteúdo de fósforo no fluido fetal. A concentração de fósforo em $\mathrm{g} / \mathrm{kg}$ apresentou um elevado grau de instabilidade de sua média, o que impede qualquer afirmação a seu respeito.

O conteúdo de cálcio e de fósforo do útero gestante foi afetado pelo número de fetos, porém não $\mathrm{o}$ foi pelo manejo nutricional aplicado (Tab. 2), sendo que o feto respondeu por $90 \%$ e $98 \%$ do conteúdo de cálcio e de fósforo do útero gestante, respectivamente.

A composição de cálcio e de fósforo da glândula mamária foi afetada pela gestação (Tab. 2 e 3 ), entretanto o número de fetos gestados não afetou sua composição (Tab. 2). As diferenças observadas são atribuídas à idade gestacional vazia ou gestante. $O$ conteúdo de cálcio no útero das vazias foi de $0,9 \mathrm{~g}$, e este valor passou para 3 a 4,4 gramas na glândula mamária aos 140 dias. Este aumento no conteúdo de quase cinco vezes é atribuído à presença de colostro na glândula mamária das ovelhas nesta idade. 
Souza et al.

Tabela 3. Conteúdo de cálcio e de fósforo do útero gestante de ovelhas Santa Inês

\begin{tabular}{|c|c|c|c|c|c|c|c|c|c|}
\hline & \multicolumn{2}{|c|}{ Manejo } & \multicolumn{3}{|c|}{ Número de fetos } & \multicolumn{3}{|c|}{ Efeito } & \multirow{2}{*}{$\begin{array}{c}\text { CV } \\
\%\end{array}$} \\
\hline & $\mathrm{R}$ & NR & 0 feto & 1 feto & 2 fetos & $\mathrm{PN}$ & NF & INT & \\
\hline \multicolumn{10}{|c|}{ Útero e membranas } \\
\hline $\mathrm{Ca}(\mathrm{g} / \mathrm{kg})$ & 0,492 & 0,443 & $0,200 b$ & $0,656 \mathrm{a}$ & $0,548 \mathrm{a}$ & NS & $*$ & $*$ & 21,11 \\
\hline $\mathrm{Ca}(\mathrm{g})$ & 0,73 & 0,70 & $0,018 b$ & $0,83 \mathrm{a}$ & $1,00 \mathrm{a}$ & NS & $*$ & NS & 33,91 \\
\hline $\mathrm{P}(\mathrm{g} / \mathrm{kg}$ & 0,542 & 0,450 & 0,480 & 0,510 & 0,497 & $*$ & NS & NS & 16,39 \\
\hline$P(g)$ & 0,81 & 0,71 & $0,046 \mathrm{c}$ & $0,64 b$ & $0,89 a$ & NS & $*$ & NS & 25,10 \\
\hline \multicolumn{10}{|l|}{ Feto } \\
\hline $\mathrm{Ca}(\mathrm{g} / \mathrm{kg})$ & 14,66 & 14,34 & & 14,82 & 14,18 & NS & NS & NS & 27,69 \\
\hline $\mathrm{Ca}(\mathrm{g})$ & 69,74 & 76,55 & & $57,86 \mathrm{~b}$ & $88,43 a$ & NS & $*$ & NS & 32,29 \\
\hline $\mathrm{P}(\mathrm{g} / \mathrm{kg}$ & 7,59 & 7,36 & & 7,62 & 7,32 & NS & NS & NS & 17,95 \\
\hline$P(g)$ & 36,03 & 39,14 & & $29,25 b$ & $45,92 \mathrm{a}$ & NS & $*$ & NS & 21,43 \\
\hline \multicolumn{10}{|l|}{ Fluido fetal } \\
\hline $\mathrm{Ca}(\mathrm{g} / \mathrm{kg})$ & 1,58 & 2,26 & & 2,15 & 1,69 & NS & NS & NS & 54,71 \\
\hline $\mathrm{Ca}(\mathrm{g})$ & 0,107 & 0,117 & & $0,070 \mathrm{~b}$ & $0,153 \mathrm{a}$ & NS & $*$ & NS & 42,15 \\
\hline $\mathrm{P}(\mathrm{g} / \mathrm{kg}$ & 0,032 & 0,071 & & 0,080 & 0,022 & NS & NS & NS & 117,5 \\
\hline$P(g)$ & 0,034 & 0,047 & & 0,045 & 0,037 & NS & NS & NS & 58,45 \\
\hline \multicolumn{10}{|c|}{ Útero gestante $^{1}$} \\
\hline $\mathrm{Ca}(\mathrm{g} / \mathrm{kg})$ & 9,35 & 9,29 & & 9,67 & 9,13 & NS & NS & NS & 34,28 \\
\hline $\mathrm{Ca}(\mathrm{g})$ & 67,97 & 68,18 & & $57,55 b$ & $89,19 \mathrm{a}$ & NS & NS & NS & 39,15 \\
\hline $\mathrm{P}(\mathrm{g} / \mathrm{kg}$ & 5,14 & 5,01 & & 5,36 & 4,80 & NS & NS & NS & 19,35 \\
\hline$P(g)$ & 37,36 & 36,77 & & $30,31 b$ & $47,61 \mathrm{a}$ & NS & $*$ & NS & 21,16 \\
\hline \multicolumn{10}{|c|}{ Glândula mamária } \\
\hline $\mathrm{Ca}(\mathrm{g} / \mathrm{kg})$ & 4,25 & 3,5 & $0,78 b$ & $6,25 a$ & $4,60 \mathrm{a}$ & NS & $*$ & NS & 51,17 \\
\hline $\mathrm{Ca}(\mathrm{g})$ & 3,79 & 2,11 & $0,900 \mathrm{~b}$ & $4,34 \mathrm{a}$ & $4,42 \mathrm{a}$ & NS & $*$ & NS & 81,50 \\
\hline $\mathrm{P}(\mathrm{g} / \mathrm{kg}$ & $4,37 \mathrm{~b}$ & $8,39 a$ & $6,10 b$ & $6,85 a$ & $6,21 \mathrm{a}$ & NS & $*$ & NS & 4,8 \\
\hline$P(g)$ & 3,90 & 4,24 & $1,92 b$ & $4,63 \mathrm{a}$ & $5,66 a$ & $\mathrm{NS}$ & $*$ & NS & 6,61 \\
\hline
\end{tabular}

*diferença significativa a $\mathrm{P}<0,05,{ }^{1}$ Soma do conteúdo de cálcio e de fósforo do útero, membranas e fluidos fetais, respectivamente; $\mathrm{NS}=$ não significativo; $\mathrm{R}=$ restrito, $\mathrm{NR}=$ não restrito.

\section{CONCLUSÕES}

O conteúdo de cálcio e de fósforo do útero gestante não é afetado pelo manejo nutricional ou pelo tipo de gestação, embora a análise individual de seus componentes mostre alterações necessárias ao equilíbrio do útero como um todo. É possível predizer o conteúdo de cálcio e de fósforo do feto por meio de equações de regressão com elevado grau de confiança.

\section{REFERÊNCIAS}

AGRICULTURAL AND FOOD RESEARCH COUNCIL - AFRC. Technical committee on responses to nutrients, Report 6 . A reappraisal of the calcium and phosphorous requirements of sheep and cattle. Nut. Abs. Rev., v.61, p.576-612, 1991

ASSOCIATION of Official Agriculture Chemicals AOAC. Official Method of Analysis. 10th Ed., Washington, DC, 1985 .
ALEXANDER, D.P.; NIXON, D.A.; WIDDAS, W.F. et al. Gestational variations in the composition of the foetal fluids and foetal urine in the sheep. J. Physiol., v.140, p.1-13, 1958.

GRACE, N.D.; WATKINSON, J.H.; MARTINSON, P.L. Accumulation of minerals by the foetus(es) and conceptus of single and twin bearing ewes. New Z. J. Agricult. Res., v.29, p.207-222, 1986.

KELLY, R.W. Nutrition and placental development. Proceedings of the Nutrition Society of Australia., p.203-210, 1992.

MACEDO JUNIOR, G.L. Exigências nutricionais de ovelhas gestantes da raça Santa Inês. 2008. 291f. tese (Doutorado em zootecnia) - Escola de Veterinária, Universidade Federal de Minas Gerais, Belo Horizonte.

MEXIA, A.L.; MACEO, F.A.F.; ALCALDE, C.R. et al. Desempenhos reprodutivo e produtivo de ovelhas Santa Inês. Rev. Bras. Zootec.,v.33, p.658-667, 2004. 
NUTRIENT requirement of ruminant livestock ARC. Commonwealth Agricultural Bureau, 2. ed., 1980.

NUTRIENT requirements of domesticated ruminants - CSIRO. Austrália: CSIRO publishing, 2007.

NUTRIENT requeriment of small ruminants NRC, Washington, D.C, National Academic Press, 2007.

OCAK, S.; EMSEN, E.; KÖYCEGIZ, F. et al. Comparasion of placental traits and their relation to litter size and parity weight in sheep. J. Anim. Sci., v.87, p.3196-3201, 2009.
PEARSON, R.A.; MELLOR, D.J. Changes in fetal fluid composition during the last 60 days of gestation in goats. J. Reprod. Fert.,v.50,p.171$173,1977$.

QUESADA, M.; McMANUS, C.; COUTO, F.A.D. Efeitos genéticos e fenotípicos sobre características de produção e reprodução de ovinos deslanados no distrito federal. Rev. Bras. Zootec., v.31, p.342-349, 2002.

ROCHA, L.P.; FRAGA, A.B.; ARAÚJO FILHO, J.T. et al. Desempenho de cordeiros cruzados em Alagoas, Brasil. Arch. Zootec., v.58, p.145-148, 2009. 